\title{
Coherent query scheme for wireless backscatter communication systems with single tag
}

\author{
Aminolah Hasanvand ${ }^{1}$, Ali Khaleghi ${ }^{1,2,3^{*}}$ (D) and Ilangko Balasingham ${ }^{2}$
}

\begin{abstract}
An un-coded multi-transmitter query scheme is introduced for wireless backscatter communication systems in which $M$ transmitters and $N$ receivers are used for single-tag connectivity $(M \times 1 \times N)$. The main idea is to harden the wireless communication channel with a tag device for high data rate readings. The proposed method is designed for multipath fading channels in which the backscatter channel is a multiplicative Rayleigh. A coherent transmit query scheme is used to increase the tag-reflected signals and simultaneously alter the fading statistics in the forward path by implementing a receiver feedback. Full-diversity performance and array gain is achieved using the receiver diversity without requiring any tag antenna diversity. Therefore, the tag device remains simple. Mathematical expressions for the probability density function (PDF) of the backscatter channel are presented using closed-form equations. Bit error rate (BER) simulations for the binary phase shift keying (BPSK) modulation are computed numerically. Diversity gain of $10 \mathrm{~dB}$ is obtained by using a $2 \times 1 \times 1$ scheme. The results show that the transmit diversity for single-tag usage performs the same as the tag antenna diversity, at the expense of a moderate transmitter complexity. The tag device remains intact as a requirement for the simplicity and size constraints. Also, the system realization becomes more feasible due to the available space on the transmitter side to accomplish the uncorrelated forward channel conditions. The feasibility study is demonstrated using softwaredefined radio (SDR) implementations.
\end{abstract}

Keywords: Backscatter communication, Transmit diversity, Antenna diversity, Wireless communication, Bit error rate, Backscatter channel feedback

\section{Introduction}

Radio frequency identification (RFID) is known in the commercial sectors. Passive or semi-passive tag devices are used in RFID to modulate the radiated RF carrier signals in the wave propagation channel, in which the reader can demodulate the tag's specific data [1]. The wireless communication approach avoids using an active transmitter in the tag device for power and space savings. By including a sensory data source in the tag device, real-time sensing and data transmission become feasible for applications in the wireless sensor network (WSN) [2-4]. An innovative implementation is for data-intensive applications such as

\footnotetext{
* Correspondence: Ali.khaleghi@ntnu.no

${ }^{1}$ K. N. Toosi University of Technology (KNTU), Tehran, Iran

${ }^{2}$ Norwegian University of Science and Technology (NTNU), Trondheim, Norway

Full list of author information is available at the end of the article
}

video streaming using the semi-passive devices. The importance is that the power associated with the transmitter task can be eliminated from the tag device. This permits cost, space, and power saving for semi-passive devices for guaranteed longevity with the available power resources. It is also possible to use the ambient signals from radio stations for the backscatter communications; the ambient signals are used to illuminate the tag device instead of the reader's transmitter [5-7]. Considering high data rate communication for data-intensive applications, we target hardening the communication channel with a semi-passive tag device in the multipath fading channels.

Backscatter communication is a radar approach that is different from one-way wireless communication in which the wave propagation channel observes two distinct paths. In the forward path, the RF carrier is emitted from the transmitter and has no information. In the return path, 
the tag device modulates the tag's antenna impedance that appeared as a variable radar cross section (RCS) [8]. The backward channel includes the tag-specific information in the form of amplitude or phase modulation of the radiated carrier. The remote receiver decodes the tag antenna reflections and extracts the tag's data [9]. The modulation level is determined by the number of the loads used in the tag device for switching among [10]. Thus, ultra-low power tag becomes feasible thanks to using an ultra-low power switch device instead of an active transmitter with the power-hungry frontend. The communication link performance can be calculated using Radar equations.

One specific environment for the backscatter application is an indoor quasi-stationary channel. The channel can be modeled as a slow fading multipath environment. By assuming the modulation bandwidth, which is the tag antenna switching speed, less than the coherence bandwidth of the backward channel, the frequency selectivity of the channel is neglected. Thus, a Rayleigh channel in the forward and backward paths is realized for the non-line of sight (NLOS) scenarios. Considering independent channels in the forward and backward links, which can be attained by separating the transmitter and the receiver antennas within a distance more than the spatial correlation of the channel, the cascade channel becomes a multiplication of two independent Rayleigh channels. The equivalent channel is no longer Rayleigh [11], and it observes deeper fading conditions. To establish a reliable link, the statistics of the multiplicative Rayleigh channel should be positively altered in a way to convert it back to a Rayleigh or Gaussian channel. Thus, the channel can be hardened for high data rate connectivity. Antenna diversity is a practical approach that can be implemented in the data path, i.e., tag or receiver antenna diversity $[12,13]$.

\subsection{Related works}

The application of antenna diversity in the receiver side has been demonstrated $[14,15]$. The diversity gain is marginal because the forward channel is always Rayleigh, and the overall channel is the multiplication of Rayleigh and diversity combined channel. Tag antenna diversity has been proposed to combat fading in the forward channel [16]. The requirement is that the tag antennas should be physically spaced with large distances, more than the spatial correlation of the channel. The minimum distance for uncorrelated channels depends on the wave angle of arrivals (AoAs) and is about half a wavelength for uniformly distributed AoAs. The correlation distance is larger if this condition cannot be attained. Therefore, full diversity gain fails to achieve in a compact tag device despite the simple structure. Further, using multiple tag antennas requires multiple and synchronous electronic switch circuits to alter the antenna impedances simultaneously over all the antennas that complicate the tag system.
Using multiple transmitters does not influence the statistics of the multiplicative channels $[11,17,18]$, because the underlying assumption is that the transmitter is not the information source. The only achievement with using multiple transmitter schemes is a reduced spatial correlation distance by increasing the AoAs. The multi-transmitting effect in backscatter communication has been studied in $[19,20]$ as a unitary query method of transmission with tag-specific space-time coding (STC). This method is a breakthrough and provides a significant gain by using time diversity in the forward channel besides the spatial diversity (STC coding) in the backward channel and outperforms the uniform query method of transmission. The drawback is the increased tag complexity and power consumption in addition to the sophisticated transmitter scheme that should be operated in a synchronous manner with the tag device for improved performance. Another form of the multi-transmitting scheme is conducted in [21, 22], where multi-sin transmitting scheme (frequency diversity) is used to transfer more power to the tag device to achieve better performance in the reading range.

\subsection{Motivation and contributions}

In this paper, we propose an un-coded transmit diversity scheme using a minor feedback from the receiver to improve the BER (bit error rate) performance and increase the communication range by altering the overall channel statistics. We call it "minor" because only the received signal strength is used to establish coherency in the query transmitters. The receiver feedback is feasible because the transceivers are closely located, and the received signal strength can be accessed and used in the transmitter for optimal system operation. The proposed transmit diversity performs the same as tag antenna diversity [11], but with a single-tag antenna. The system performance is better than the unitary query method of transmission [19] without added complexity in the tag device. Our approach is optimum for single-tag usage. Furthermore, it can provide time reversal spatial focusing at the tag location for a targeted data reading. The main contributions of this paper are as follows:

- Transmit diversity with equal gain combining (EGC) is proposed at the query end with channel feedback of one receiver for intended tag connectivity. It is shown that the probability density function (PDF) and BER performances can be improved significantly with the main complexity at the reader side.

- The analytical expressions for PDF of the channel for a single tag are derived followed by BER performance calculations for binary phase shift keying (BPSK) modulation. The receiver diversity is added to show the BER performance. 
- Demonstration of the transmitter query scheme is shown using software defined radio implementations.

\subsection{Organization}

The paper is organized as follows. In Section 2, the mathematical expressions for the statistics of the backscatter communication channel are presented. The analytical expressions for the statistics of the proposed transmit query scheme with feedback channel are calculated in Section 3. Numerical simulation results for BER performance of the transmit query for BPSK modulation are given in Section 4. Experimental validation of the approach for single-channel implementation using a software-defined radio (SDR) is given in Section 5. In Section 6, the receiver diversity is added to the proposed backscatter system in which we show that the feedback from one receiver is sufficient to achieve full-diversity performance. Section 8 concludes this paper.

\section{Backscatter communications}

Any multiple input multiple output (MIMO) backscatter communication system consists of three operational parts: query end, tag end, and receiving end [19]. Figure 1 shows the scheme of a backscatter communication system in a single-tag scenario. An unmodulated continues wave $(\mathrm{CW})$ signal is transmitted in the wave propagation channel. The signal paths that experience tag reflections carry tag-specific data. The signal paths that do not experience the tag device are considered as stationary interference. At the receiving end, the interference signal is removed and the signal demodulation is conducted. The backscatter channel can be interpreted as a pinhole channel [23], and the only difference is that the data is generated at the tag (pinhole) instead of the transmitter.
A nominal backscatter communication setup uses a single-transmitter antenna and multiple receiver antennas, or it can include multiple antennas in the tag device. Using multiple transmitter antennas does not influence the communication system performance [11, 17, 24].

Consider a $M \times 1 \times N$ backscatter communication system with $M$ transmitters in the query end, single-tag antenna and $\mathrm{N}$ receivers are shown in Fig. 2. Assume that the modulation bandwidth generated by the tag switching is less than the coherence bandwidth of the backward channel. Thus, the frequency selectivity of the channel is disregarded, and inter-symbol interference (ISI) is neglected. Therefore, the channel model in the forward and backward paths is expressed as Rayleigh. As shown in Fig. 2, the transmitted carrier signals are expressed as $X$, the forward channel is defined by $\mathbf{H}^{\mathbf{f}}$ in which $h_{i}^{f}=\left|h_{i}^{f}\right| e^{j<h_{i}^{f} \in \mathbb{C}}$ are the channel coefficients. Superposition of these signals in the tag antenna is modulated by the tag data signal $s$ (exp. $s \in\{-1,1\}$ for BPSK modulation). The backward channel, $\mathbf{H}^{b}$, is expressed by its coefficients, $h_{i}^{b}=\mid h_{i}^{b}$ $\mid e^{j<h_{i}^{b} \in \mathbb{C}}$. The received signal is corrupted by the noise $\mathbf{N}\left(n_{i}=\left|n_{i}\right| e^{\left.j<n_{i} \in \mathbb{C}\right)}\right.$ at the $i$-th receiver.

The transmitted signals might be received at the receivers without experiencing the tag path. The addition of these direct coupling signals is considered as a stationary interference. Considering the narrowband signaling in the multipath channel, the channel becomes flat fading, and the received signal is expressed as:

$$
\mathbf{Y}_{\mathbf{N} \times \mathbf{1}}=\mathbf{X}_{\mathbf{1} \times \mathbf{M}} \mathbf{H}_{\mathbf{M} \times \mathbf{1}}^{\mathrm{f}} \mathbf{H}_{\mathbf{N} \times \mathbf{1}}^{\mathrm{b}} S+\mathbf{N}_{\mathbf{N} \times \mathbf{1}}+\mathbf{D}_{\mathbf{N} \times \mathbf{1}}
$$

where $\mathbf{D}$ is the direct coupling and

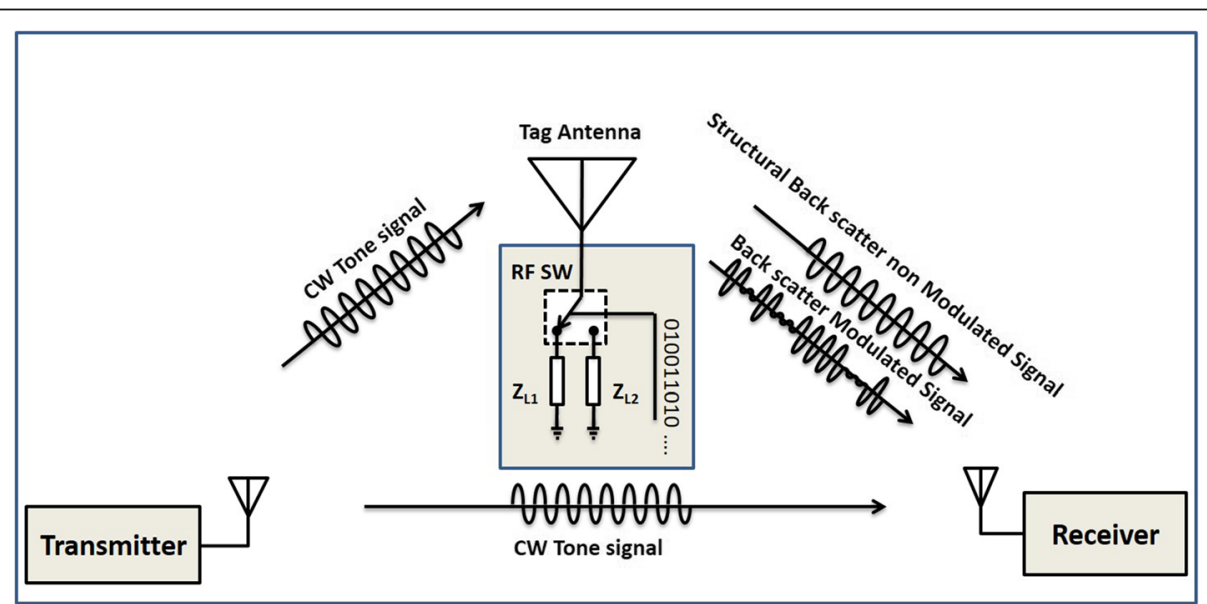

Fig. 1 Illustration of backscatter communication system. The data alters differential radar cross section $(\Delta \mathrm{RCS})$ of the tag device that results in backscatter signal modulation 


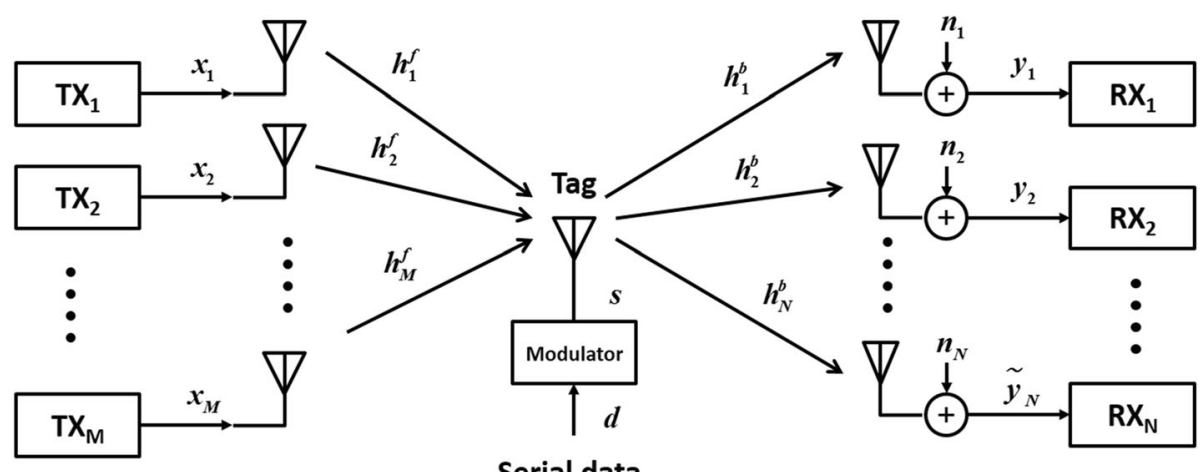

Serial data

Fig. 2 Scheme of a conventional $M \times 1 \times N$ cascaded backscatter channel

$$
\begin{gathered}
\mathbf{x}=\left[\begin{array}{ll}
x_{1} & x_{2} \ldots x_{M}
\end{array}\right] \quad, \quad x_{i}=A_{i} e^{j \phi_{i}}, i=1,2, \ldots, M \\
\mathbf{H}^{\mathbf{f}}=\left[\begin{array}{c}
h_{1}^{f} \\
h_{2}^{f} \\
\vdots \\
h_{M}^{f}
\end{array}\right], \quad \mathbf{H}^{\mathbf{b}}=\left[\begin{array}{c}
h_{1}^{b} \\
h_{2}^{b} \\
M \\
h_{N}^{b}
\end{array}\right], \quad \mathbf{N}=\left[\begin{array}{c}
n_{1} \\
n_{2} \\
\vdots \\
n_{N}
\end{array}\right]
\end{gathered}
$$

The term $\mathbf{D}_{\mathbf{N} \times \mathbf{1}}$ can be eliminated after signal demodulation by applying proper filtering for removing the stationary signals. This term consists of a tag structural radar cross section (RCS) and environment clutters that do not include the tag's information $[8,25]$. For a stationary channel, $\mathbf{D}_{\mathbf{N} \times \mathbf{1}}$ appeared as a DC component in the demodulator and a simple high-pass filter can remove it.

By considering the constant and equally distributed transmission power for the query end, i.e., $\left|x_{i}\right|=A_{i}$ $=\frac{1}{\sqrt{M}}, i=1,2, \ldots, M$, the received signal for the $i$-th receiver becomes

$$
y_{i}=K\left(\frac{1}{\sqrt{M}} \sum_{k=1}^{M} h_{k}^{f} \cdot e^{j \phi_{k}}\right) h_{i}^{b} s+\hat{n}_{i}
$$

where $K$ is a deterministic scalar coefficient including the filtering gain, transmitting signal amplitude, and other gains and $\hat{n}_{i}$ is the color Gaussian noise.

For a single antenna in transmission, i.e., $1 \times 1 \times N$, the received signal statistics at the $i$-th receiver is proportional to the production of two complex i.i.d Gaussian variables $h_{1}^{f}$ and $h_{i}^{b}$ as follows,

$$
y_{i}=K\left(h_{1}^{f} \cdot e^{j \phi_{1}}\right) h_{i}^{b} s+\hat{n}_{i} \propto h_{1}^{f} \cdot h_{i}^{b}
$$

Thus, the envelope of the received signal has PDF of two Rayleigh products.
Considering the $M$ transmitters, i.e., $M \times 1 \times N$, the superposition of the carriers at the tag antenna are modulated and then reflected in the channel. Therefore, the received signal is written as

$$
\begin{aligned}
& y_{i}{ }^{\propto}\left(\sum_{k=1}^{M} h_{k}^{f} \cdot e^{i \phi_{k}}\right) h_{i}^{b}=\left(h_{1}^{f} \cdot e^{j \phi_{1}}+h_{2}^{f} \cdot e^{j \phi_{2}}+\cdots+h_{M}^{f} \cdot e^{j \phi_{M}}\right) \\
& h_{i}^{b}=z_{i}^{f} \times h_{i}^{b}
\end{aligned}
$$

For flat fading channels with i.i.d. complex Gaussian property, the linear combination of $\mathrm{M}$ channels is i.i.d. complex Gaussian variable. The envelope of these channels is Rayleigh, and the received signal in the $i$-th receiver has PDF of two Rayleigh products [26]. Therefore, the above multi-transmitter system has no improvement compared to a single-transmitter scheme [17].

\section{Multi-antenna transmission with channel feedback}

Under the condition that the transmitter signals are coherently summed at the tag antenna, the maximum amount of the backscatter signal is achieved at the receiver. We show how this combination alters the channel statistics. For this purpose, the forward channel must be maximized at the tag's position. Consequently, the overall gain of the forward channel should be maximized,

$$
\begin{aligned}
& \text { if } \max _{a_{k}}\left|\sum_{k=1}^{M} h_{k}^{f} \cdot e^{j \phi_{k}}\right|, \alpha_{k}=\measuredangle h_{k}^{f}+\phi_{k} \text { results : } \\
& \left\{\forall m, n=1,2, \ldots, M \mid \alpha_{m}=\alpha_{n}=\alpha\right\}
\end{aligned}
$$

To obtain the condition above, the transmitters must be synchronized with a single reference clock (see Fig. 3). 


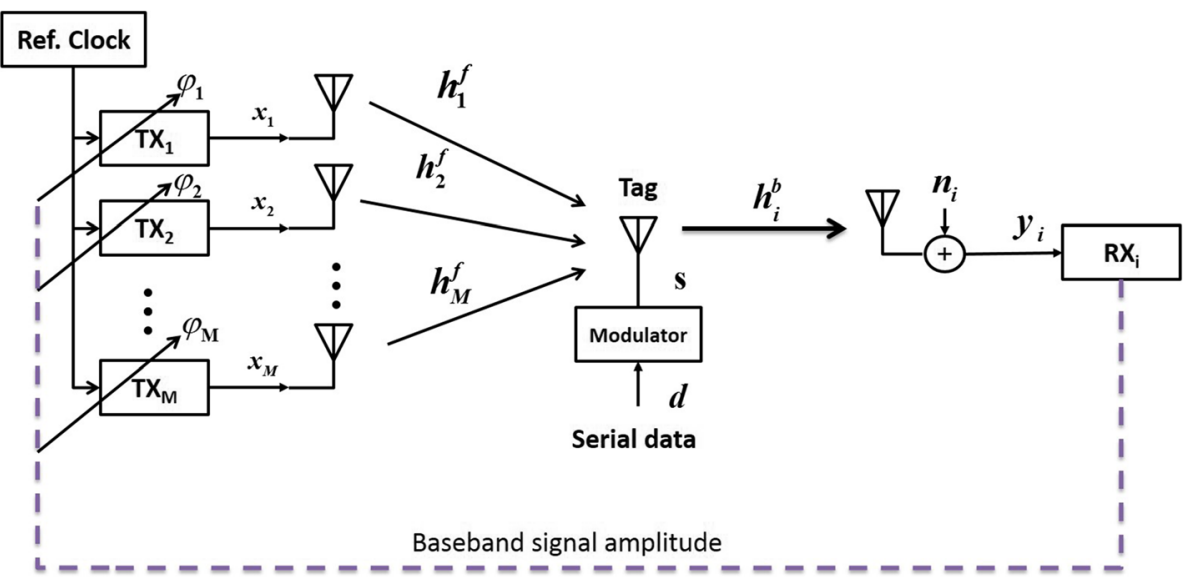

Fig. 3 Illustration of $M \times 1 \times 1$ backscatter communication system with a common reference clock. The dashed line shows feedback implementation for coherent focusing of signals at the tag location

Also, it is necessary to alter the phase of each transmitter to achieve the co-phase summation (maximize the amplitude of the received signal) at the tag location. Therefore, applying the condition in (5) to (4) results to

$$
\begin{aligned}
& y_{i}(t) \propto\left(\sum_{k=1}^{M} h_{k}^{f} \times e^{j \phi_{k}}\right) h_{i}^{b}=\left(\sum_{k=1}^{M}\left|h_{k}^{f}\right|\right) e^{j \alpha} \times\left|h_{i}^{b}\right| e^{\complement} h_{i}^{b} \\
& =\left(\sum_{k=1}^{M}\left|h_{k}^{f} \| h_{i}^{b}\right|\right) e^{j\left(\alpha+\measuredangle h_{i}^{\mathrm{b}}\right)}
\end{aligned}
$$

As a result, from (6), the PDF of the received signal envelope is the sum of M Rayleigh products. The signals terminated to the tag in the forward and backward channels may be correlated. The amount of the correlation, $\rho$, depends on the distance between the transmitter and the receiver antennas.

The elements of the forward and backscatter links can be written in the Cartesian form, respectively, as $h^{f}$ $=u^{f}+j v^{f}$ and $h^{b}=u^{b}+j v^{b}$ where $u^{f, b}$ and $v^{f, b}$ are zero-mean Gaussian random variables, $\sim \mathrm{N}\left(\mathbf{0}, \sigma_{f, b}^{2} / 2\right)$, in which $\sigma_{f}^{2}$ and $\sigma_{b}^{2}$ are the variance of $h_{k}^{f}$ and $h_{i}^{b}$, respectively. The envelope PDF of $M \times 1 \times N$ cascaded backscatter channel is derived from that of the product of two Rayleigh random variables. The closed-form PDF for dependent and independent Rayleigh products is given in [26]. Therefore, by considering random variable $Z$ as the multiplication of $\left|h_{k}^{f}\right|$ and $\left|h_{i}^{b}\right|$, i.e., $Z$ $=\left|h_{k}^{f}\right| \times\left|h_{i}^{b}\right|$, its closed-form PDF is expressed as [26]:

$$
\begin{gathered}
f_{\mathrm{z}}(\mathrm{z}, \rho)=\frac{4 z\left(1-|\rho|^{2}\right)}{\sigma_{b}^{2} \sigma_{f}^{2} \gamma^{2}} \mathrm{I}_{0}\left(\frac{2 z|\rho|}{\sigma_{b} \sigma_{f} \gamma}\right) \mathrm{K}_{0}\left(\frac{2 z}{\sigma_{b} \sigma_{f} \gamma}\right), \\
\gamma=\left(1-\rho^{2}\right), \mathrm{z} \geq 0
\end{gathered}
$$

where $\mathrm{I}_{0}$ is a zero-order, modified Bessel function of the first kind and $K_{0}$ is a zero-order, modified Bessel function of the second kind. The characteristic function, $\Phi($.$) , of$ the sum of i.i.d. random variables, $R=\sum_{k=1}^{M}\left|h_{k}^{f} \| h_{i}^{b}\right|$ (the inter parentheses of (6) that is envelop of the received signal in $i$-th receiver), is the product of their individual characteristic functions (CF), and consequently, their joint PDF can be derived by using the inverse Hankel transform. The CF (7) is found using Hankel transform [27], which was raised to the power of $\mathrm{M}$ to yield the CF for the cascaded backscatter channel of $M \times 1 \times 1$ system:

$$
\begin{aligned}
\Phi(\omega ; \rho) & =\left[\frac{\sigma_{b}^{4} \sigma_{f}^{4}}{16} \frac{\gamma^{4}}{\left(1-|\rho|^{2}\right)^{2}}\left(\omega^{2}+\frac{4(|\rho|-1)^{2}}{\sigma_{b}^{4} \sigma_{f}^{4} \gamma^{2}}\right)\right. \\
& \left.\times\left(\omega^{2}+\frac{4(|\rho|+1)^{2}}{\sigma_{b}^{4} \sigma_{f}^{4} \gamma^{2}}\right)\right]
\end{aligned}
$$

By using the inverse Hankel transform on (8), the PDF of $M \times 1 \times 1$ channel is derivable. However, it is difficult to solve its related integral analytically. Therefore, two special cases are considered for $\rho=0$, in which $h_{k}^{f}$ and $h_{i}^{b}$ are independent, and $\rho=1$, where they are completely correlated. 
The former case can be achieved by separating the receiver and the transmitter which is known as bistatic topology. The latter is known as monostatic topology where the receiver and transmitter are co-located. The analytical calculations of the PDF for bistatic and monostatic models as upper and lower limits of dependency of the forward and the backward channels are conducted. Considering the bistatic topology $(\rho=0)$ the CF becomes

$$
\Phi(\omega ; 0)=\left[\frac{\sigma_{b}^{2} \sigma_{f}^{2}}{4} \omega^{2}+1\right]^{-M}
$$

Applying the inverse Hankel transform to (9) yields the PDF of $M \times 1 \times 1$ topology as

$$
f_{r}(r, \rho=0)=r^{M}\left(\frac{2}{\sigma_{b} \sigma_{f}}\right)^{1+M} \frac{2^{1-M}}{\Gamma(M)} K_{(1-M)}\left(\frac{2 r}{\sigma_{b} \sigma_{f}}\right)
$$

where $K_{(1-M)}($.$) is the modified Bessel function of the$ order of $(1-\mathrm{M})$ and the second kind, $\Gamma($.$) , is the gamma$ function and $r$ is the channel envelope.
By considering the monostatic topology $(\rho=1)$, the CF is derived as

$$
\Phi(\omega ; \rho)=\left[\sigma_{b}^{2} \sigma_{f}^{2} \omega^{2}+1\right]^{-M / 2}
$$

Applying the inverse Hankel transform to (11) yields the PDF of $M \times 1 \times 1$ topology as

$$
f_{\mathrm{r}}(\mathrm{r}, \rho=1)=\mathrm{r}^{M / 2}\left(\frac{1}{\sigma_{b} \sigma_{f}}\right)^{1+M / 2} \frac{2^{1-M / 2}}{\Gamma(M / 2)} \mathrm{K}_{\left({ }_{1-} M / 2\right)}\left(\frac{\mathrm{r}}{\sigma_{b} \sigma_{f}}\right)
$$

The PDF of the envelope of the channel at the $n$-th receiver antenna based on the above analytical expressions is plotted in Fig. 4a.

Monte-Carlo channel realizations are provided to compute the PDF numerically. For this purpose, $10^{6}$ realizations are conducted, and the PDF of the envelope is obtained for the bistatic and monostatic topologies for $M \times 1 \times 1(M=1,2,3,4)$. Figure $4 \mathrm{~b}$ shows the numerical

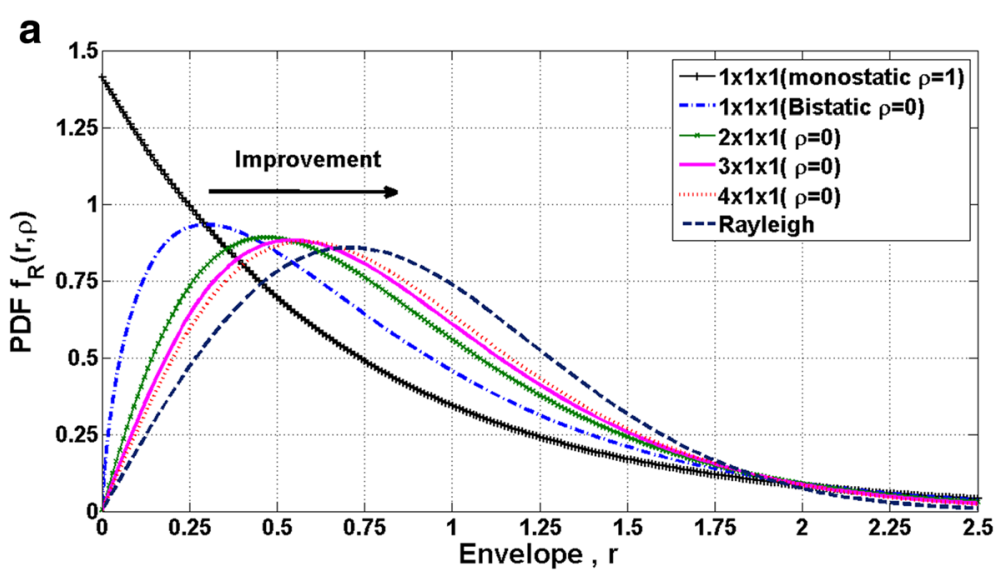

b

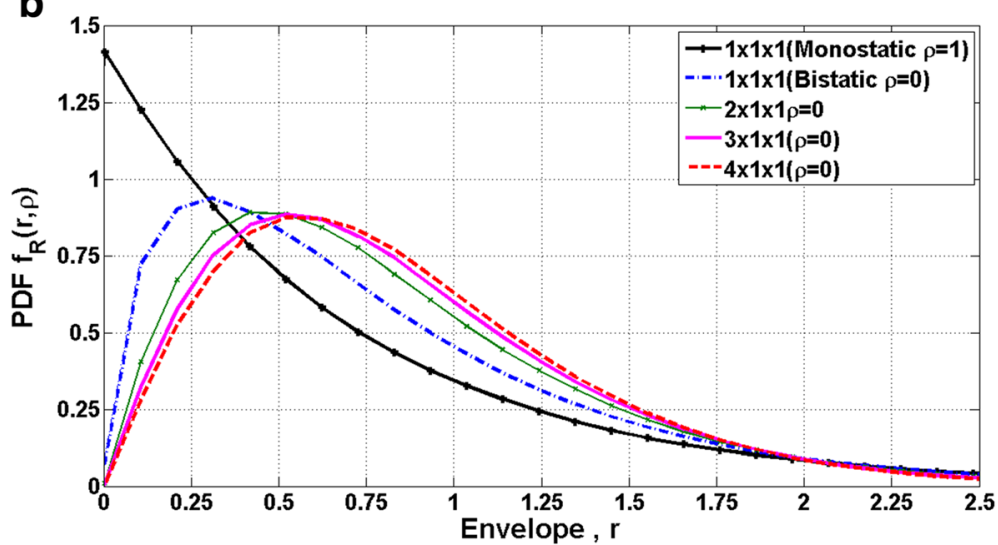

Fig. 4 PDF of backscatter channel by increasing the number of co-phased TX transmitters. a PDF results from analytical expressions for $M=1,2$, 3,4 transmitters with the proposed phase adjustment and coherent transmitter (the PDF for monostatic, bistatic, and Rayleigh are also illustrated for comparison). $\mathbf{b}$ The numerical results of PDF with channel realization 
simulation results. As shown, the results of the simulation and the above analytical calculations are in complete agreement. The PDF of a conventional one-way Rayleigh fading channel is also illustrated. We note that the PDF's are normalized to unit transmit power. Deep fading in the PDF of the cascaded backscatter channel can be seen in Fig. 4 compared to the one-way Rayleigh channel, as it is shown from the left to right arrow. By increasing the number of the transmit antennas, the corresponding envelope PDF shifts to the right indicating reduced fading probability. The most significant change is seen in the transition from a fully correlated channel, where the PDF changes from an exponential distribution of the monostatic channel to a product of Rayleigh distribution for the bistatic channel with $M$ transmitters. By increasing $\mathrm{M}$, the PDF curves approach the Rayleigh distribution, in which a Rayleigh fading can express the cascade channel for massive numbers of coherent transmitters.

The backscatter communication performance is simulated by assuming BPSK modulation with additive white Gaussian noise (AWGN). Coherent detection is used to evaluate BER performance. The number of the random channel realizations is $10^{6}$ to achieve the ensemble average of BER. In the simulations with $M$ transmitter antennas, the total power is normalized to unity. Figure 5 a shows the calculated BER versus SNR $(\mathrm{dB})$. As shown, by increasing the number of transmit antennas and by applying the phase compensation at the transmitter side, given in (10), the BER curves shift toward the left side of the graph. For instance, by

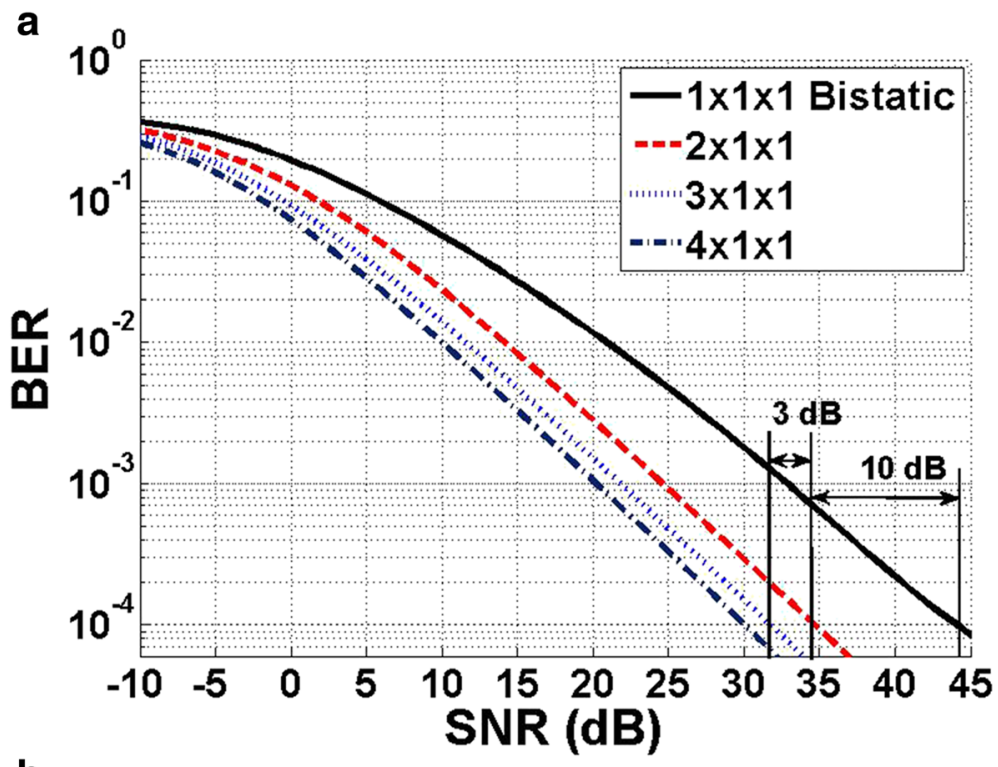

b

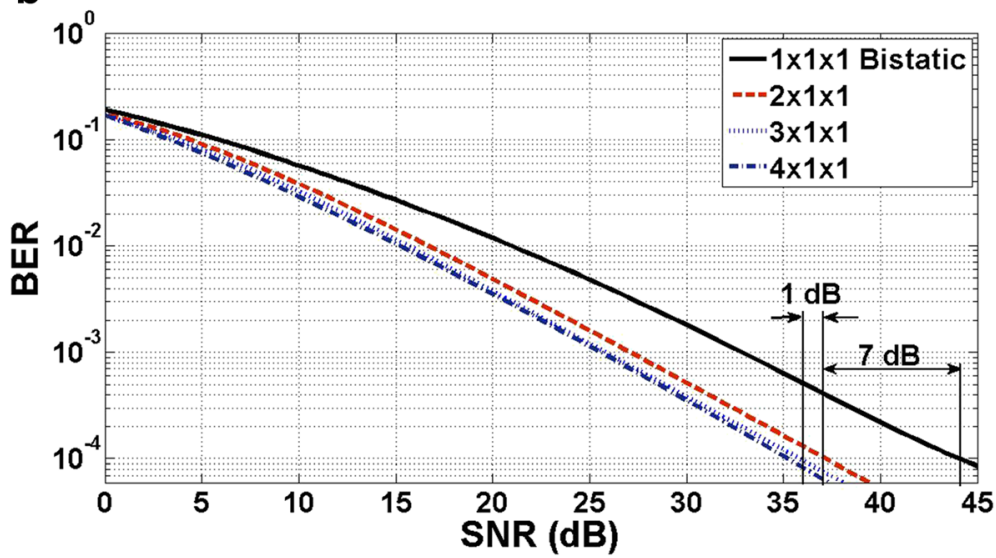

Fig. 5 Average BER curves for cascaded backscatter channels with independent, Rayleigh fading in forward and backscatter links. Un-coded BPSK modulation with coherent detection in the presence of AWGN noise is applied. Each curve represents the average BER of the signal at the $n$-th receiver with and without diversity. a Normalized total transmitted power with $M$ transmitters (diversity gain and antenna array gain are represented). b Normalized BER to constant EIRP (diversity gain due to the altered channel statistics is illustrated) 
applying two transmitter antennas, we can observe a gain of $10 \mathrm{~dB}$ for BER level $10^{-4}$ compared to a single antenna in transmission. The gain increases for $M=3$ and 4. The improvement in BER performance can be described by the transmit array gain and the modification of the channel statistics as stated in (6) and Fig. 4.

Figure $5 \mathrm{~b}$ shows the BER performance for the scenario in which the effective isotropic-radiated power (EIRP) is normalized to the unity. Therefore, the array gain is removed in BER calculations, and the improvement caused by the transmit diversity is obtained. As shown, a gain of $7 \mathrm{~dB}$ is achieved for BER level of $10^{-4}$ due to the changes in the channel statistics. The rest of the $3 \mathrm{~dB}$ gain in Fig. $5 \mathrm{a}$ is related to the antenna array gain in transmission. In an ultimate scenario, with massive numbers of antennas in the forward channel, the overall channel statistics approaches Rayleigh performance for $(M \times 1 \times 1, M \rightarrow \infty)$.

\section{Performance analysis by numerical simulations}

The implementation of the proposed transmit diversity is simple. As a result from (5), for a co-phase signal combination at the tag location, the transmitters' phase $\left(\phi_{i}, i=1,2, \ldots, M\right)$ must be adjusted. Figure 3 shows the co-phase multi-transmit backscatter communication scheme. In this implementation, all the transmitters should have common reference clock, and a feedback from one receiver is used for the phase tuning. In the phase tuning process, one of the transmitters sends continues wave $(\mathrm{CW})$ signal in the channel. The tag reflects its data in the backscatter channel, and the reflections are received and demodulated by the receiver system. The signal level at the receiver is monitored. The second transmitter starts the operation by transmitting CW signal, and the phase of the transmitted carrier is swept in the range $[-\pi, \pi]$ to obtain the maximum data signal at the receiver side. This process is similar to the phasor summation of the wave vectors. The procedure is continued for the consequent transmitters, and the phase tuning is performed for the newly added transmitter. Therefore, a relative phase among the transmitters are selected that increase SNR at the receiver. The proposed co-phasing algorithm is a simple approach, and it may be replaced by other fast and more adaptive algorithms which are not considered in this work.

According to the approach above, the phase of each transmitter must be tuned to add SNR in the receiver. Therefore, the sensitivity of the scheme relative to the phase deviation among the transmitters is studied. Figure 6 shows the simulated average BER performance for $2 \times 1 \times$ 1 backscatter channel with independent forward and backward Rayleigh fading channels. As shown, the phase deviation of $0^{\circ}$ provides the best BER performance, in which the two transmitter signals are coherently added at the tag location. By the phase deviation from the optimal value, the performance is degraded. However, the performance is almost intact for the phase deviations up to $120^{\circ}$. The reason is the vector summation of the complex Gaussian random variables. From $120^{\circ}$ to $170^{\circ}$, the performance is degraded rapidly in which the system performance is similar to a single antenna in transmission (see Fig. 6). In case that the phase error lies in the range of $170^{\circ}-180^{\circ}$, the system performance is worse than the single antenna configuration. In this case, the signals at the tag are destructive. Therefore, the probability to worsen the performance with dual antenna compared to the single antenna in transmission is about $10 / 180$ or $5.5 \%$. This result shows that if the second transmitter comes to the operation, with a random phase value, there is a probability of $5.5 \%$ that the system performance is degraded. However, with the phase sweeping, the system performance can be improved for a wide range of phase values for instance $66 \%$ (i.e., 120/180). Therefore, the most essential characteristic of co-phase multi-transmit in backscatter communication is its low sensitivity to the phase deviation from the optimal value.

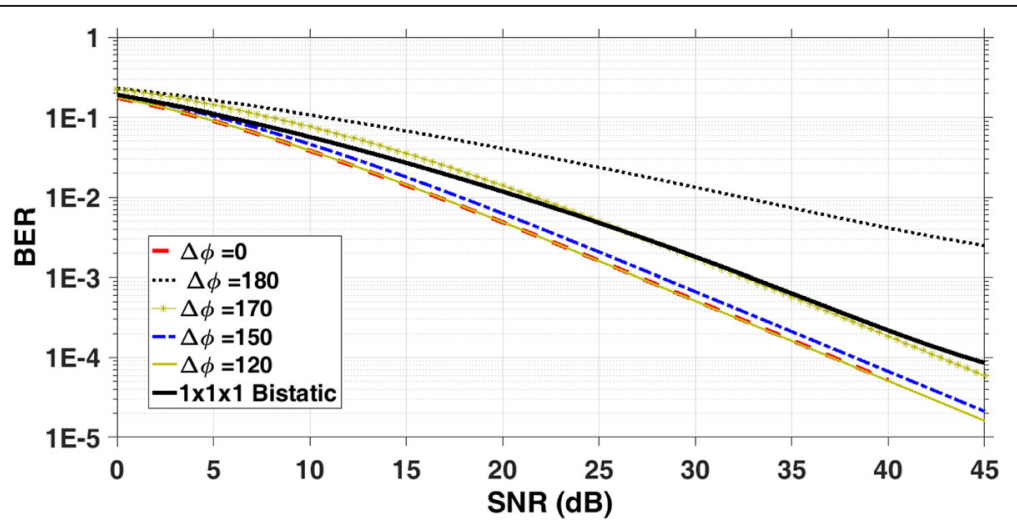

Fig. 6 BER versus SNR for phase deviation $(\Delta \varphi$, in degrees) from the optimal value $(\Delta \varphi=0)$ for a system with two query transmitters $(2 \times 1 \times 1)$. The system performance is less sensitive to phase miss alignments up to $120^{\circ}$ 


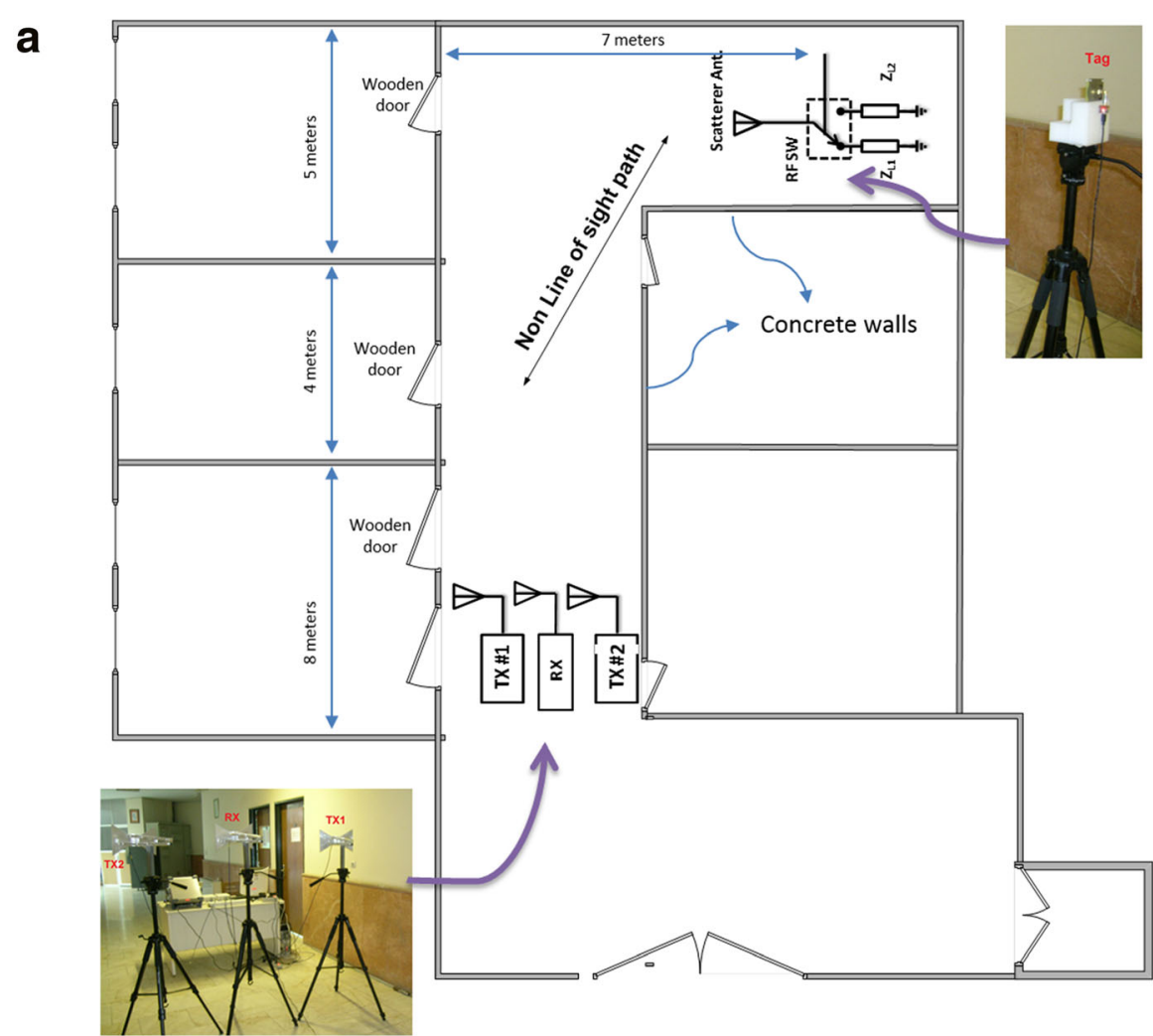

b

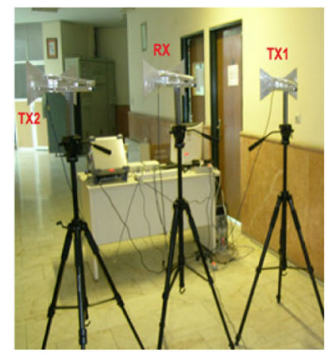

C

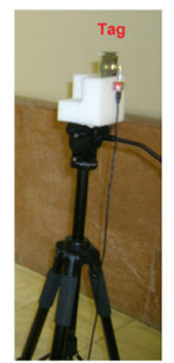

d

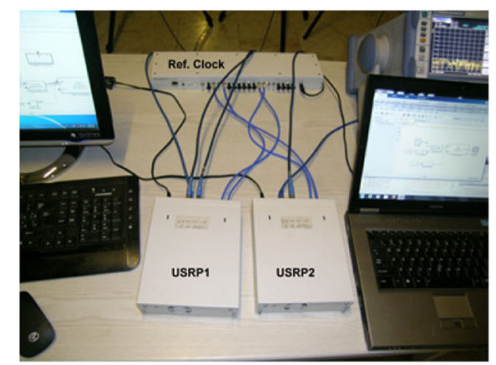

Fig. 7 Backscatter implementation setup using two transmitter query scheme a The plan of the indoor measurement environment, $\mathbf{b}$ two transmit query and receiving antennas, $\mathbf{c}$ tag antenna $\mathbf{d}$ transmitter and receiver radios based on USRP N210 modules and clock reference Octoclock-G

\section{Experimental results}

Demonstration of multi-transmitting coherent query scheme for backscatter communication is conducted using the experimental setup shown in Fig. 7 . The plan of the indoor environment is illustrated in Fig. 7a. The transmitter antennas are log-periodic and the tag antenna is a circular patch on a ground plane with resonance frequency at $2.5 \mathrm{GHz}$ (Fig. 7b, c). The tag antenna port is connected to an RF switchboard which is controlled by the data source from a computer program. The tag reflection is maximized by self-grounding the antenna (resonance mode) and is minimized by open circuit condition.

The transmitter antennas are located in one corridor and transmit $\mathrm{CW}$ signal at $2.5 \mathrm{GHz}$. To generate NLOS conditions, the tag antenna is placed in the second corridor to remove the possible direct path from the transmitters. Two transmitters and one receiver are used for wireless communication with the semi-passive tag device. The transceivers are realized using software-defined radio (SDR), USRP N210. Two SDR transmitters are synchronized by using an external clock source Octoclock-G (Fig. 8d). Therefore, the relative phase of the transmitter carriers can be tuned via the system's software program. One of the SDRs is also used as the receiver. MATLAB Simulink is used to implement the baseband processing functions. All SDRs are connected to their host PCs through Giga Ethernet ensuring enough baseband bandwidth. 

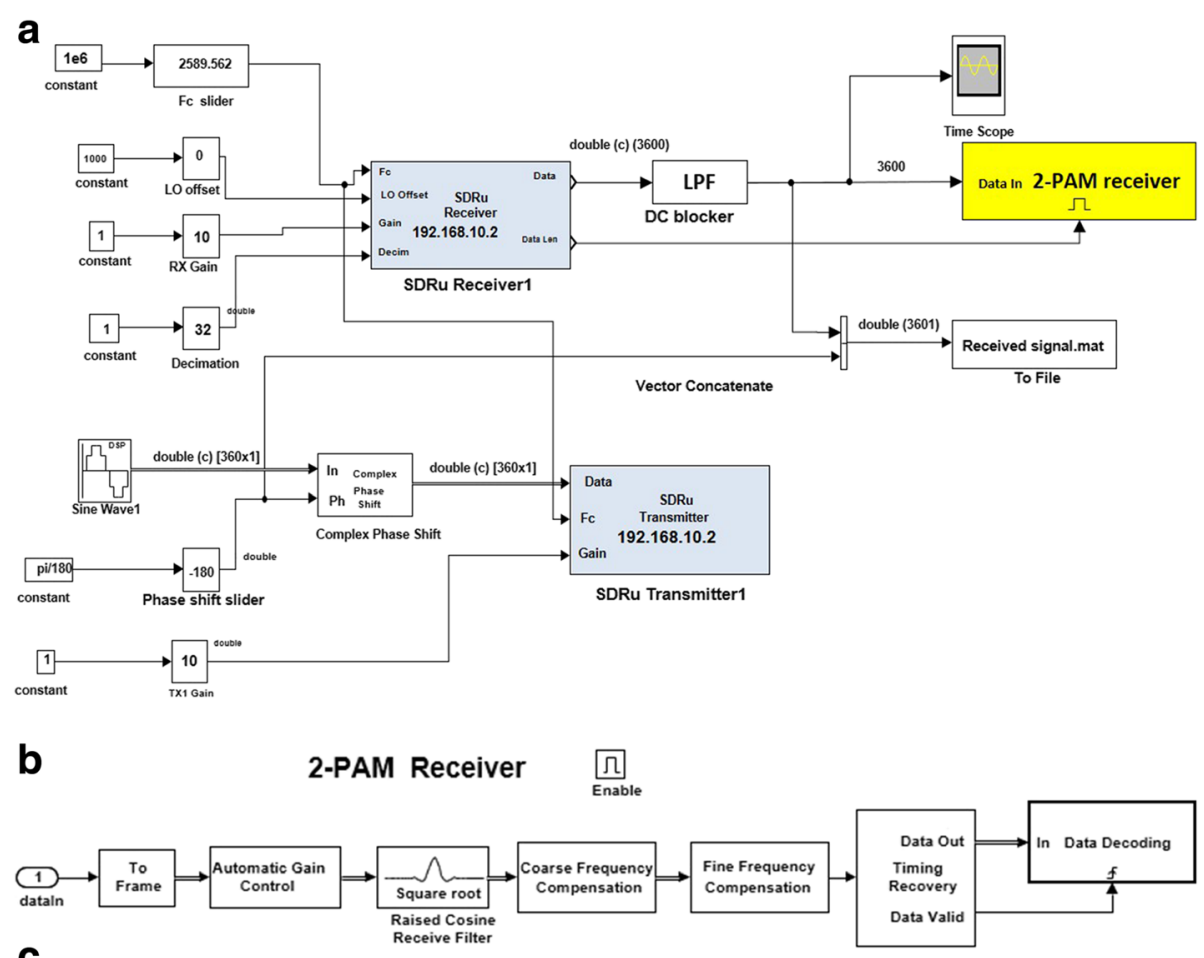

C
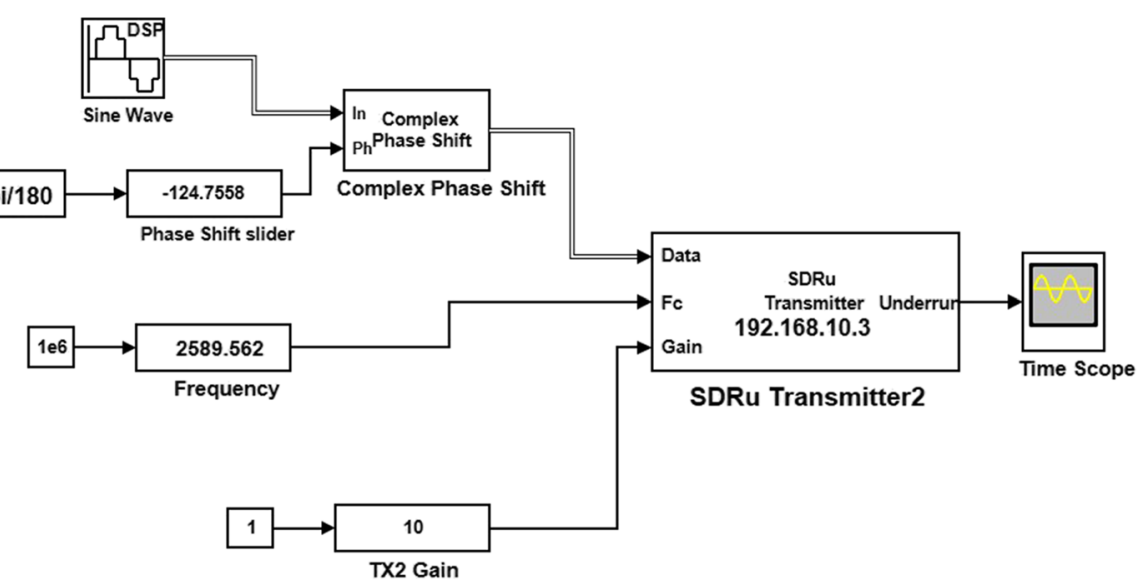

Fig. 8 Matlab Simulink block diagram for the backscatter M-query scheme using USRP N210 a implemented USRP No.1 as the transmitter and receiver $\mathbf{b}$ 2-PAM non-coherent receiver chain for the backscattered data extraction c transmitter module USRP No.2 with phase tunning feature

The tag switch is controlled by the data from a PC which transfers the content of an image data in a defined protocol. All the requirements for data extraction in the receiver side are considered in this protocol.

In the experiment, we consider constant net power in the query ends. Thus, in the dual transmitter scheme, the transmitted power for each radio is reduced by $3 \mathrm{~dB}$ compared to the single transmitter. Figure 8 shows the block diagram of the realized $2 \times$ $1 \times 1$ backscatter communication system using USRP N210 and the related MATLAB Simulink target. The transmitter part can be tuned to operate in a defined carrier frequency. The gain of the transmitters can be adjusted independently. In addition, a phase shifter is used that can modify the relative phase of the synchronized transmitters. The receiver includes automatic gain control (AGC), receiver match filter, coarse and fine frequency compensator, clock recovery, and data decoding blocks. Figure 9a shows the baseband received signal detected by the SDR receiver; the eye diagram for both in-phase and quadrature components is shown in Fig. 9b. The overall system reads the transmitted image data via the backscatter link.

To demonstrate the applicability of the coherent query scheme, the detected backscatter signal level by 
a

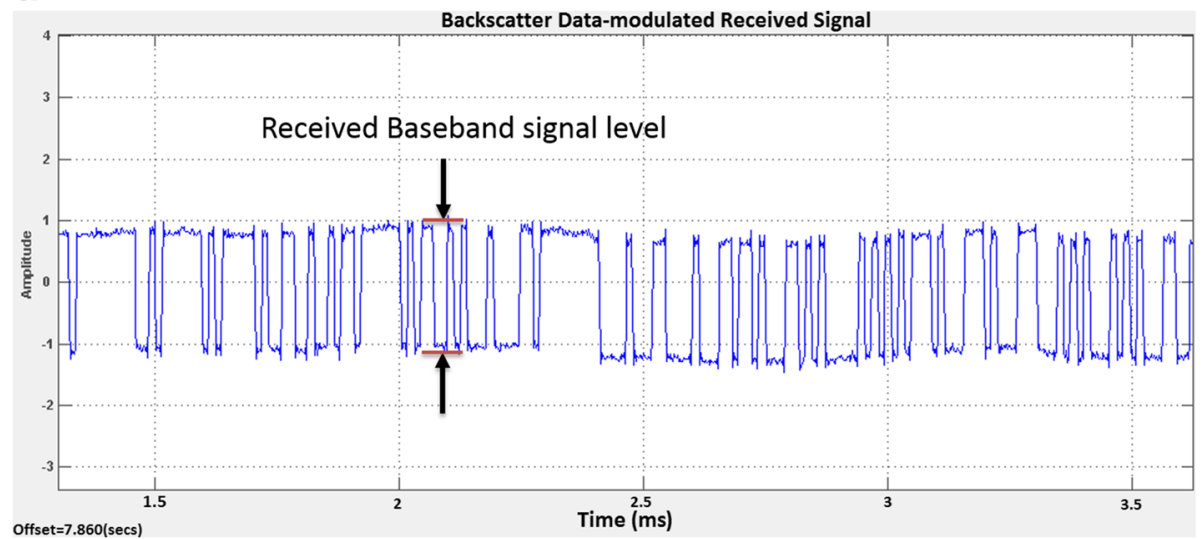

b
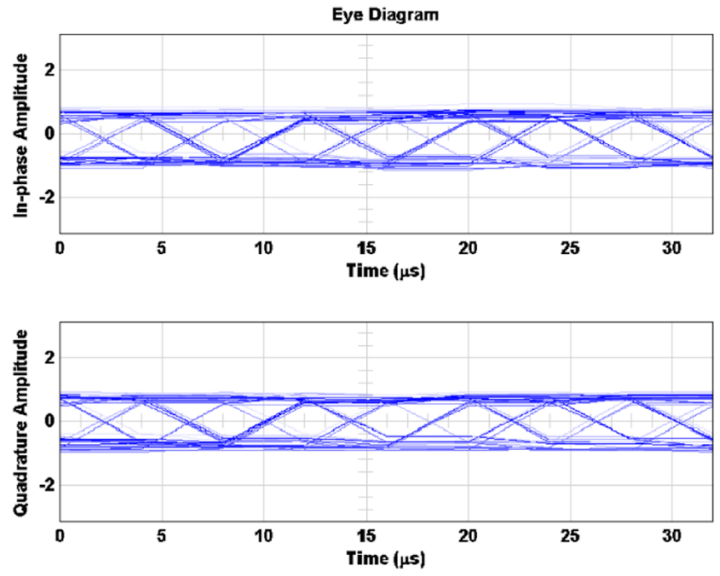

Fig. 9 Experimental received signal a Baseband received signal after removing the direct coupling term $\mathbf{b}$ eye diagram after clock recovery for I \& Q channels

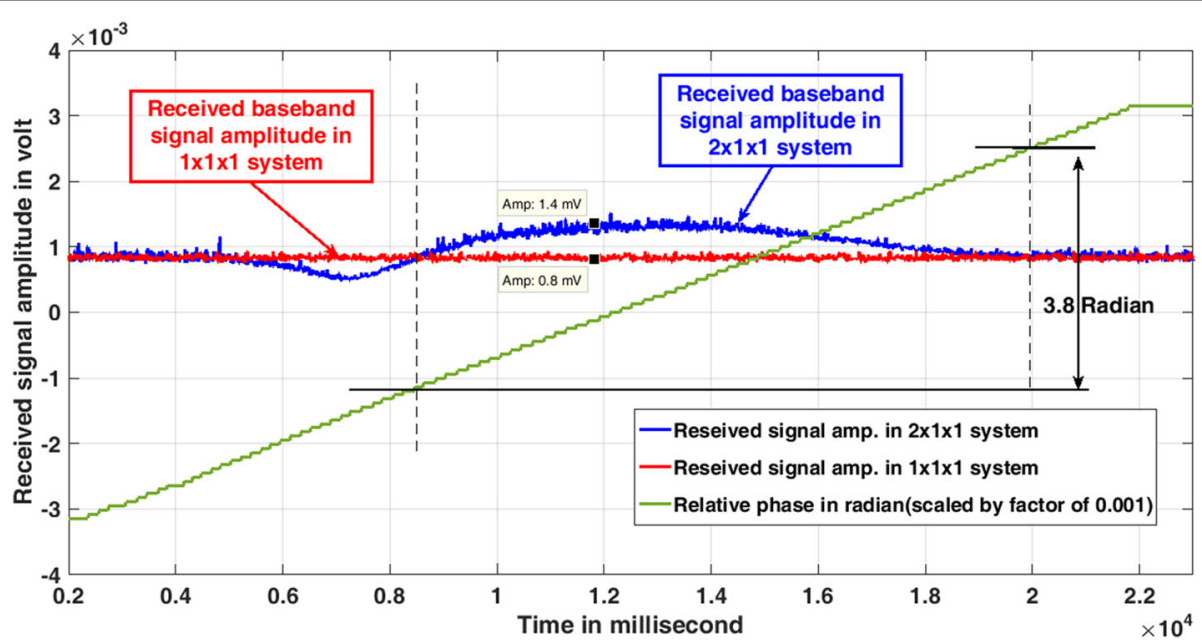

Fig. 10 Measured data signal amplitude after demodulation using one-transmitter query $1 \times 1 \times 1$ (red curve) and two transmitter query $2 \times 1 \times 1$ (blue curve) with relative phase variations 
the SDR receiver is used as the basis for the performance evaluation (Fig. 9a). MATLAB Simulink is used for the real-time monitoring of the tag data in the receiver. First, one SDR transmitter with constant power is applied. Figure 10 shows the level of the detected signal for one-transmitter query system in the quasi-static channel. Using a two-transmitter scheme, by assigning half of the power to each transmitter, the received signal is detected. By sweeping the phase of one transmitter in the range $[-\pi, \pi]$ with $5^{\circ}$ steps per second, we can observe the received signal as the blue curve. As shown, the detected backscatter signal level is increased from 0.8 to $1.4 \mathrm{mv}$ which is about $5 \mathrm{~dB}$ in terms of the received signal power. Also, for $60 \%$ of the phase interval, the baseband signal amplitude using $2 \times 1 \times 1$ scheme is above the $1 \times 1 \times$ 1 scheme and confirms the simulation results in Section 4.

The reason for the improved signal level at the receiver is EM wave focusing in the forward path. To assess this fact, a spectrum analyzer is connected to the tag antenna port, and the received signal level is monitored by sweeping the transmitter phase. By monitoring the received carrier signal power at the tag antenna, it is observed that the transmitted signals with the above defined optimum phase values are coherently summed at the tag antenna location.

\section{Receiver diversity}

Receiver diversity is applied to further improve the BER performance by altering the channel statistics in the backward path. For this purpose, we use $\mathrm{M}$-transmit and $\mathrm{N}$-receive antennas. In the implementation procedure, feedback from one receiver is applied to the transmitter chain and the phase tuning task is completed to assure the coherent summation of the forwarding paths at the tag location. Thus, the transmit diversity task is completed. The receiver diversity is applied to coherently combine the data signals in the receivers. To determine the amount of the improvement, Monte-Carlo simulations is conducted for $10^{6}$ channel realizations, and EGC is used as the signal combining method. Figure 11 shows the results of the simulations. The average BER curves for BPSK modulation are plotted for $2 \times 1 \times 2,2 \times 1 \times$ 3 and $3 \times 1 \times 3$ schemes. The BER curves for the multiplicative Rayleigh, Rayleigh, and AWGN are also illustrated. As shown, by using $2 \times 1 \times 2$ configuration, the BER performance for a level of $10^{-4}$ is improved by $19 \mathrm{~dB}$ compared to the non-diversity $(1 \times 1 \times 1)$ backscatter system. By using three transceivers $(3 \times$ $1 \times 3$ ), the improvement is $4.5 \mathrm{~dB}$ compared to the dual transceivers. In an ultimate state with massive transceivers, the system performance approaches AWGN channel.

\section{Methods}

A coherent query scheme is proposed for backscatter wireless communication with a single-tag device in a Rayleigh multipath channel. The proposed method uses the receiver feedback for providing appropriate phase information in the query end and thus spatial wave focusing in the tag antenna. Using the proposed method, the multiplicative Rayleigh fading channel statistics is altered in favor of BER. An analytical approach is used to derive the fading channel statistics, and Monte-Carlo numerical simulations confirm the results. The BER performance is calculated numerically for $\mathrm{M}$ - transmitter scheme with the channel feedback. The provided method shifts the tag device complexity to the transmitter side in which a moderate complexity is acceptable. The approach is validated experimentally by using SDR implementations. Two transmitters in the query end are synchronized by using a common clock distributor network, and feedback from one receiver is used to control the

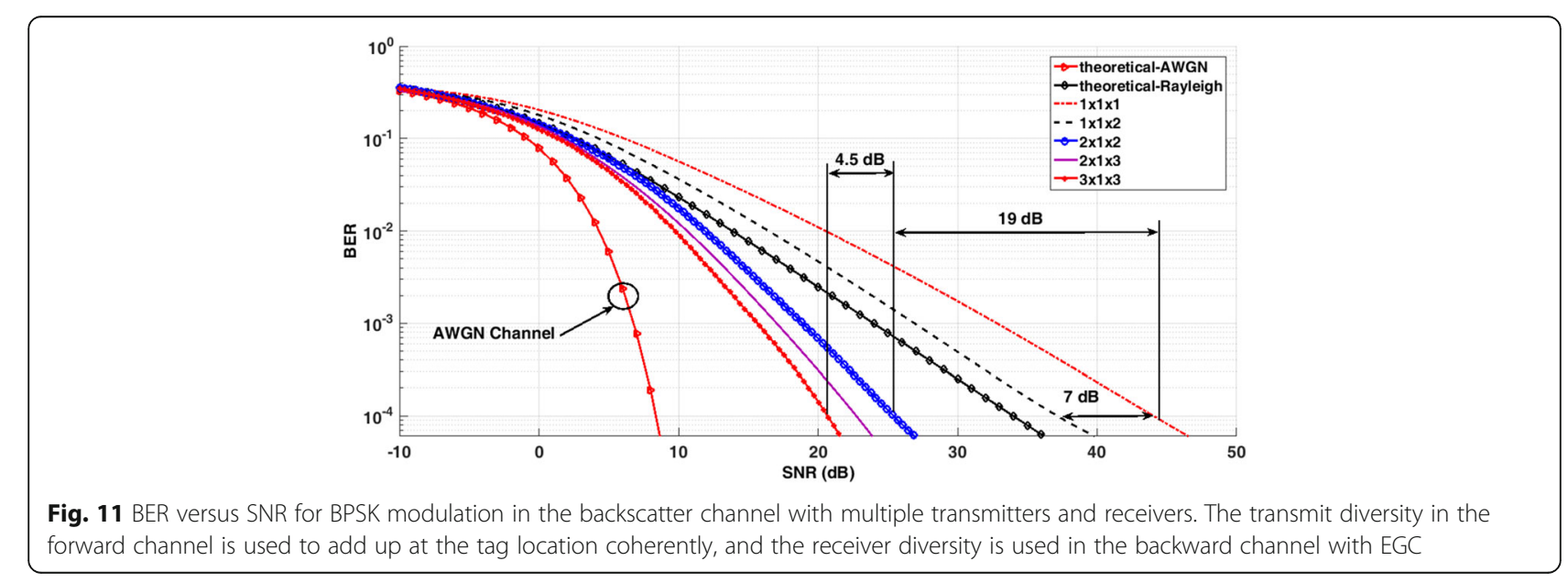


phase difference between the transmitter carrier signals. Details of system implementation using MATLAB Simulink are provided, and the backscatter communication with a data-intensive tag device is demonstrated. Measurements show coherent summation of the transmitted signal in the tag location, using the coherent query scheme, that results improved data connectivity in multipath channels.

\section{Conclusions}

Transmit diversity is proposed for backscatter wireless communication with a single-tag antenna. The primary objective is to combat deep fading in the backscatter channel for possible high data rate communication with a tag device. It is shown that using coherent query antennas on the reader side, with a feedback channel from one receiver, the overall backscatter link can be improved by $10 \mathrm{~dB}$ for $\mathrm{BER}=10^{-4}$ and BPSK modulation using two transmitter antennas. The improvement is caused by the coherent summation of the multipath signals in the forward path at the tag location, thanks to the receiver feedback. The channel statistics are positively modified by using multiple coherent and phase-tuned transmitters, in which the equivalent channel statistics can be converted from multiplicative Rayleigh to a Rayleigh channel by increasing the number of the phase-tuned transmitters. The transmit array gain adds further to the system performance. The analytical expressions of the channel statistics are provided. It is also indicated that the transmit diversity performance is less sensitive to the phase deviation among the transmitters. Therefore, there is a broad range of relative phase values that a backscatter system with multiple transmitters can operate optimally. The system implementation is demonstrated by experimental measurements in an indoor channel.

Using receive diversity in addition to the transmit diversity is recommended to improve the channel statistics and obtain better BER performance than in a Rayleigh channel. In the case of receiver diversity, only a feedback from one receiver in the chain can be used for tuning the phase differences among the transmitters. The diversity combining at the receiver can be applied following the forward channel focusing that improves the BER performance significantly.

\footnotetext{
Abbreviations

AGC: Automatic Gain Control; AWGN: Additive white Gaussian noise; BER: Bit error rate; BPSK: Binary phase shift keying; CW: Continues wave; EGC: Equal gain combining; EIRP: Effective isotropic radiated power; ISI: Inter-symbol interference; MIMO: Multiple input multiple output; NLOS: Non-line of sight; PDF: Probability density function; RCS: Radar cross section; RFID: Radio frequency identification; SDR: Software-defined radio; STC: Space-time coding; WSN: Wireless sensor network
}

\section{Acknowledgements}

The authors would like to thank Wireless Test Terminal Lab (WTTL), KNTU, for the help and support of the experiments. Also, the Department of Electronic Systems at NTNU and the Research Council of Norway under the project WINNOW grant no. 270957/O70 for supporting the visiting scholar.

\section{Authors' contributions}

The authors have contributed jointly to all parts on the preparation of this manuscript, and all authors read and approved the final manuscript.

\section{Authors' information}

Aminolah Hasanvand is a Ph.D. student at K. N. Toosi University of Technology, Tehran, Iran. Ali Khaleghi is an adjunct professor at K. N. Toosi University of Technology and is a scientist at the Norwegian University of Technology (NTNU), Trondheim, Norway. llangko Balasingham is a professor at the Norwegian University of Science and Technology (NTNU), Trondheim, Norway.

Ethics approval and consent to participate

Not applicable.

Consent for publication

Not applicable.

\section{Competing interests}

The authors declare that they have no competing interests.

\section{Publisher's Note}

Springer Nature remains neutral with regard to jurisdictional claims in published maps and institutional affiliations.

\section{Author details}

${ }^{1} \mathrm{~K}$. N. Toosi University of Technology (KNTU), Tehran, Iran. ${ }^{2}$ Norwegian University of Science and Technology (NTNU), Trondheim, Norway. ${ }^{3}$ Signal Processing Group, Department of Electronic Systems, Norwegian University of Science and Technology, Electro Building, NTNU, N-7491 Trondheim, Norway.

Received: 3 November 2017 Accepted: 19 June 2018

Published online: 20 July 2018

References

1. D M Dobkin, The Rf in RFID: Uhf RFID in Practice: Newnes; 2012.

2. S Roy, V Jandhyala, JR Smith, D Wetherall, BP Otis, R Chakraborty, M Buettner, DJ Yeager, Y-C Ko, AP Sample, RFID: from supply chains to sensor nets. Proc. IEEE 98(9), 1583-1592 (2010)

3. K Han, K Huang, Wirelessly powered backscatter communication networks: modeling, coverage, and capacity. IEEE Trans. Wirel. Commun. 16(4), 25482561 (2017)

4. C Psomas, I Krikidis, Backscatter communications for wireless powered sensor networks with collision resolution. IEEE Wireless Communications Letters 6(5), 650-653 (2017)

5. G Wang, F Gao, R Fan, C Tellambura, Ambient backscatter communication systems: detection and performance analysis. IEEE Trans. Commun. 64(11), 4836-4846 (2016)

6. X Zhou, G Wang, Y Wang, J Cheng, An approximate BER analysis for ambient backscatter communication systems with tag selection. IEEE Access 5, 22552-22558 (2017)

7. Y Liu, G Wang, Z Dou, Z Zhong, Coding and detection schemes for ambient backscatter communication systems. IEEE Access 5, 4947-4953 (2017)

8. P Nikitin, K Rao, R Martinez, Differential RCS of RFID tag. Electron. Lett. 43(8), 431-432 (2007)

9. D Kim, MA Ingram, WW Smith, Measurements of small-scale fading and path loss for long range RF tags. IEEE Trans. Antennas Propag. 51(8), 17401749 (2003)

10. SJ Thomas, MS Reynolds, A 96 Mbit/sec, $15.5 \mathrm{pJ} / \mathrm{bit}$ 16-QAM modulator for UHF backscatter communication. IEEE International Conference. 185-190 (2012)

11. JD Griffin, GD Durgin, Gains for RF tags using multiple antennas. IEEE Trans. Antennas Propag. 56(2), 563-570 (2008) 
12. J-S Kim, K-H Shin, S-M Park, W-K Choi, N-S Seong, Polarization and space diversity antenna using inverted-F antennas for RFID reader applications. Antennas and Wireless Propagation Letters, IEEE 5(1), 265-268 (2006)

13. MS Abouzeid, L Lopacinski, E Grass, T Kaiser, R Kraemer, Efiïcient and lowcomplexity space time code for massive MIMO RFID systems. 12th Iberian Conference on IEEE. 1-6 (2017)

14. MA Ingram, MF Demirkol, D Kim, in Proc. ISSSE. Transmit diversity and spatial multiplexing for RF links using modulated backscatter. Proceedings of the 2001 International Symposium on Signals, Systems, and Electronics (ISSSE '01). (Tokyo, 2001)

15. A Rahmati, L Zhong, M Hiltunen, R Jana, Reliability techniques for RFID-based object tracking applications,(2007) pp. 113-118

16. JD Griffin, GD Durgin, Multipath fading measurements for multi-antenna backscatter RFID at $5.8 \mathrm{GHz}$. IEEE International Conference on. 322-329 (2009)

17. C Boyer, S Roy, Space time coding for backscatter RFID. IEEE Trans. Wirel. Commun. 12(5), 2272-2280 (2013)

18. C He, X Chen, ZJ Wang, W Su, On the performance of MIMO RFID backscattering channels. EURASIP J. Wirel. Commun. Netw. 2012(1), 1-15 (2012)

19. C He, ZJ Wang, VCM Leung, Unitary query for the MxLXN MIMO backscatter RFID channel. IEEE Trans. Wirel. Commun. 14(5), 2613-2625 (2015)

20. C He, ZJ Wang, C Miao, Query diversity schemes for backscatter RFID communications with single-antenna tags. IEEE Trans. Veh. Technol. 66(8), 6932-6941 (2017)

21. AJS Boaventura, NB Carvalho, The design of a high-performance multisine RFID reader. IEEE Transactions on Microwave Theory and Techniques. 65(9), 3389-3400 (2017)

22. H-C Liu, Y-F Chen, Y-T Chen, A frequency diverse Gen2 RFID system with isolated continuous wave emitters. J. Networks 2(5), 54-60 (2007)

23. D Chizhik, G Foschini, R Valenzuela, Capacities of multi-element transmit and receive antennas: correlations and keyholes. Electron. Lett. 36(13), 1 (2000)

24. C Boyer, S Roy, Invited paper-backscatter communication and RFID: coding, energy, and MIMO analysis. IEEE Trans. Commun. 62(3), 770-785 (2014)

25. D Hotte, R Siragusa, Y Duroc, S Tedjini, Radar cross-section measurement in millimetre-wave for passive millimetre-wave identification tags. IET Microwaves, Antennas \& Propagation 9(15), 1733-1739 (2015)

26. M. K. Simon, Probability distributions involving Gaussian random variables: a handbook for engineers and scientists: (Springer Science \& Business Media, New York, 2007)

27. A. D. Poularikas, Transforms and applications handbook: (CRC Press, Boca Raton, 2010).

\section{Submit your manuscript to a SpringerOpen ${ }^{\circ}$ journal and benefit from:}

- Convenient online submission

- Rigorous peer review

Open access: articles freely available online

- High visibility within the field

- Retaining the copyright to your article

Submit your next manuscript at $>$ springeropen.com 